\title{
T1/ST2 promotes T helper 2 cell activation and polyfunctionality in bronchopulmonary mycosis
}

\author{
D Piehler ${ }^{1}$, A Grahnert ${ }^{1}$, M Eschke $^{2}$, T Richter ${ }^{1}$, G Köhler $^{3}$, W Stenzel ${ }^{4}$ and G Alber ${ }^{1}$
}

Interleukin (IL)-33 enhances T helper (Th)2 immunity via its receptor T1/ST2. Infection with the yeast-like pathogen Cryptococcus neoformans is usually controlled by a Th1-mediated immune response. The mechanisms responsible for nonprotective Th2 immunity leading to allergic inflammation in pulmonary cryptococcosis are still not fully understood. Using a murine pulmonary model of $C$. neoformans infection, we report that T1/ST2 expression correlates with the intensity of Th2 activation, as demonstrated by the expression of CD25 and CD44 and downregulation of CD62L. Antigen-specific T1/ST2 ${ }^{+}$Th cells are the primary source of the Th2 cytokines IL-5 and IL-13 as compared with wildtype T1/ST2 ${ }^{-}$Th cells or Th cells from T1/ST2 ${ }^{-/}$mice. In addition, $\mathrm{T} 1 / \mathrm{ST} 2^{+}$Th cells almost exclusively contain bi- and trifunctional Th2 cytokine-producing Th cells compared with T1/ST2- Th cells or Th cells from T1/ST2-/- mice. Finally, T1/ST2-driven Th2 development resulted in defective pulmonary fungal control. These data demonstrate that T1/ST2 directs Th2 cell activation and polyfunctionality in allergic bronchopulmonary mycosis.

\section{INTRODUCTION}

The development of protective immunity against airborne fungal pathogens (e.g., Aspergillus fumigatus, Paracoccidioides brasiliensis) mainly relies on immunity mediated by T helper (Th)1 and partially Th17-associated mechanisms in the respiratory tract. ${ }^{1,2}$ In contrast, Th2-mediated immune mechanisms are often insufficient and do not contribute to control or elimination of the fungal pathogens. ${ }^{3}$ There is rather an increasing body of reports showing even enhancement of infection and development of prototypical Th2-related diseases such as asthma and allergic inflammation. ${ }^{4-6}$ Among the fungi of clinical importance, Cryptococcus neoformans gained attention, especially since the HIV pandemic as it can cause severe infection in immunocompromised patients associated with high mortality. ${ }^{7}$ As we and others showed earlier, susceptibility against $C$. neoformans is linked to Th2 cells and the Th2 cytokines interleukin (IL)-4, IL-5, and IL-13. ${ }^{8-12}$ Recently, we could show that in eosinophil-deficient mice Th2 cells, characterized by the expression of the IL-33 receptor (termed T1/ST2), are decreased, resulting in a more Th1-biased cytokine response and enhanced growth control of C. neoformans. ${ }^{13}$ Taken together, these reports indicate that pulmonary cryptococcosis is a highly suitable model to study Th2 immunity.
IL-33 is a multifunctional molecule as it can behave as a transcription factor ${ }^{14}$ as well as a mediator with Th2-associated cytokine activity affecting innate and adaptive immunity. ${ }^{15}$ This broad array of regulatory and effector mechanisms led to high priority in research in several fields of immunology and beyond that, e.g., cardiovascular disease and metabolism. ${ }^{16,17}$ The role of IL-33 in fungal infections is controversial as there are reports about either mediating protection ${ }^{18}$ or susceptibility. ${ }^{19}$ Thus, it was intriguing to investigate the role of IL-33 in pulmonary cryptococcosis and to characterize the potential role of IL-33 in Th2-driven immunopathology. The objective of this study was to analyze activation of and cytokine production by pulmonary Th cells in the presence or absence of T1/ST2. Our results demonstrate that T1/ST2 impacts Th cell activation associated with the development of disease-promoting Th2 polyfunctionality in bronchopulmonary mycosis.

\section{RESULTS}

T1/ST2 induces IL-5- and IL-13-dependent allergic inflammation during pulmonary cryptococcosis

The established features of an immunopathological Th2 response against $C$. neoformans include the recruitment of

\footnotetext{
${ }^{1}$ Institute of Immunology, College of Veterinary Medicine, University of Leipzig, Leipzig, Germany. ${ }^{2}$ Institute of Immunology and the Molecular Pathogenesis Group, Center for Biotechnology and Biomedicine, College of Veterinary Medicine, University of Leipzig, Leipzig, Germany. ${ }^{3}$ Gerhard-Domagk-Institute for Pathology, Universitätsklinikum Münster, Münster, Germany. ${ }^{4}$ Department of Neuropathology, Charité Universitätsmedizin, Berlin, Germany. Correspondence: G Alber (alber@rz.unileipzig.de) 

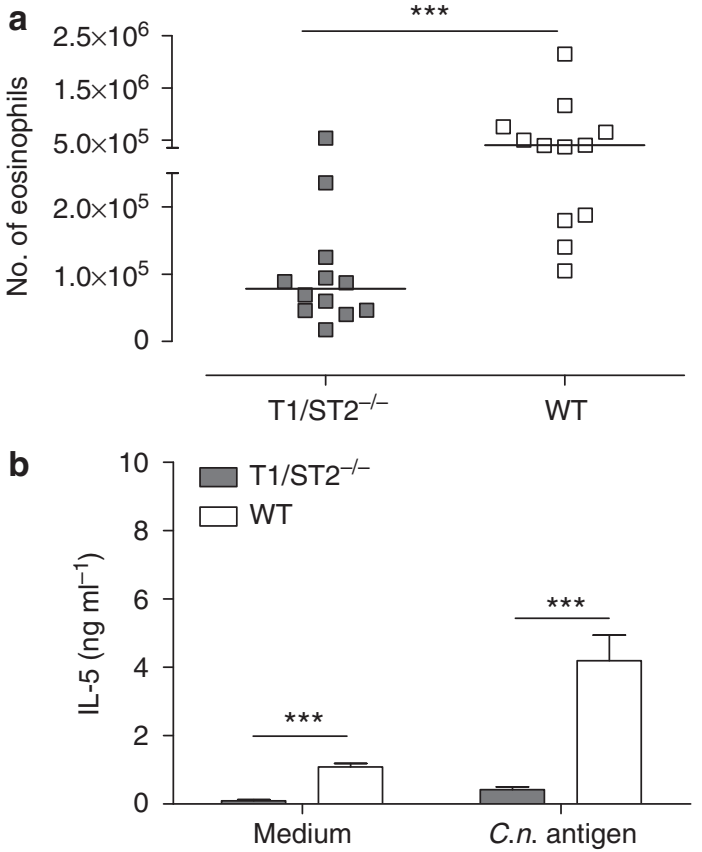

Figure 1 Interleukin (IL)-5 synthesis and pulmonary eosinophilia are tightly linked with the expression of T1/ST2. Wild-type (WT) and T1/ST2 $2^{-/-}$ mice were infected intranasally with Cryptococcus neoformans 1841 and analyzed 49 days post infection (dpi) ( $n=6$ mice per genotype and experiment). (a) Pulmonary leukocytes were isolated from individual mice, counted and stained for eosinophilic granulocytes (SSChigh, Siglec- $\mathrm{F}^{+}$, and $\mathrm{CD} 11 \mathrm{c}^{-/ \mathrm{dim}}$ ), and analyzed by flow cytometry according to the Methods section. (b) Isolated pulmonary leukocytes of two mice (of the same genotype) were pooled (i.e., 3 pools per genotype and experiment) and restimulated for $22 \mathrm{~h}$ with $C$. neoformans (C.n.) antigen or left untreated. IL-5 was measured by enzyme-linked immunosorbent assay (ELISA) in culture supernatants. Data from two independent experiments were pooled and expressed as mean \pm s.e.m. Statistical analysis was performed using the Mann-Whitney $U$-test for the analysis of eosinophilic granulocytes and the Student's $t$-test for supernatant analysis. ${ }^{* *} P \leq 0.001$.

eosinophilic granulocytes and bronchoepithelial mucus production resembling allergic inflammation. ${ }^{9,13}$ IL-33 gained special interest in allergy research for its ability to initiate and promote this kind of inflammation..$^{20}$ Therefore, we initially investigated the potential role of IL-33 in cryptococcosis by analyzing mice with a deletion of the IL-33 receptor ST2 (IL-33R-deficient mice are further termed $\mathrm{T} 1 / \mathrm{ST} 2^{-1-}$ mice) ${ }^{21}$ We analyzed wild-type (WT) and T1/ST2 ${ }^{-1-}$ mice 7 weeks after intranasal infection when WT mice harbor pulmonary Th2 cells and produce Th2associated cytokines. ${ }^{13}$ In infected T1/ST2 ${ }^{-1-}$ mice, recruitment of eosinophilic granulocytes identified by flow cytometry as $\mathrm{SSC}^{\text {high }}$, Siglec- $\mathrm{F}^{+}$, and $\mathrm{CD} 11 \mathrm{c}^{-/ \mathrm{dim}}$ is significantly reduced but not abolished in the lung (Figure 1a). In addition, analysis of periodic acid-Schiff-positive (PAS) epithelial cells (Figure 2a) reveals enhanced mucus production in WT mice upon microscopic quantification (Figure 2b). The appearance of eosinophils and induction of mucus hyperplasia in the lungs are tightly connected to the Th2-associated cytokines IL-5 and IL-13. ${ }^{22,23}$ Therefore, we analyzed if the observed reduction in eosinophils and bronchial mucus production in the absence of
T1/ST2 is the result of diminished synthesis of IL-5 and IL-13, respectively. Indeed, restimulation of pulmonary leukocytes with cryptococcal antigen revealed a strongly reduced Cryptococcusspecific production of IL-5 and IL-13 (Figures $1 \mathbf{b}$ and $2 \mathbf{c}$ ). As we have shown earlier, cytokine production upon re-stimulation with cryptococcal antigen primarily depends on Th cells. ${ }^{24}$ Therefore, IL-5- and IL-13-mediated immunopathology is dependent on T1/ST2 signaling in Th cells, although there is residual production of both cytokines (Figures $\mathbf{1 b}$ and $\mathbf{2 c}$ Medium).

\section{T1/ST2 is upregulated on Th cells upon infection with C. neoformans and drives pulmonary Th cell recruitment}

As a consequence of reduced Cryptococcus-specific IL-5 and IL-13 production, we determined the numbers of pulmonary Th cells to exclude the possibility that the observed reduction in the synthesis of IL- 5 and IL- 13 is a result of reduced Th cells in the lungs of T1/ST2 $2^{-1-}$ mice. Naïve WT and T1/ST2 ${ }^{-1-}$ mice comprise similar numbers of pulmonary Th cells (data not shown). In response to infection with $C$. neoformans, relative numbers in both groups are comparable, although WT mice in total harbor slightly more Th cells (Figure 3a), pointing to a T1/ST2dependent difference in the Th phenotype underlying allergic inflammation shown in Figures 1 and 2. Next, we had a more detailed look on the phenotype of the Th cells to analyze T1/ST2 expression during infection with C. neoformans (Figure 3b). T1/ST2 has been shown to be a marker of Th2 cells in different systems, ${ }^{25-27}$ including cryptococcosis. ${ }^{28}$ About 5-17\% (median $12.4 \%$; Figure 3c; left panel) of the Th cells in WT mice express T1/ST2 after infection, showing a clear induction of T1/ST2 on Th cells following infection and displaying a remarkable population in the lung (Figure 3c; right panel). It is of note that in other lymphoid organs, e.g., draining lymph nodes, the frequency of $\mathrm{T} 1 / \mathrm{ST} 2{ }^{+}$Th cell was much lower (data not shown). Interestingly, T1/ST2 expression was also found on eosinophilic granulocytes and alveolar macrophages (data not shown).

\section{T1/ST2 ${ }^{*}$ Th cells in pulmonary cryptococcosis represent highly activated Th cells}

There is ample evidence for T1/ST2 being a marker of differentiated Th2 cells. ${ }^{21,26,29}$ However, it is not clear which regulatory influence T1/ST2 expression has on the activation status of Th2 cells. Therefore, we compared expression of the activation markers IL-2R $\alpha$ (CD25), CD44 and CD62L in T1/ST2 ${ }^{+}$and T1/ST2 ${ }^{-}$ Th cells of infected WT mice. We found that upon infection of WT mice with C. neoformans, pulmonary T1/ST2-expressing Th cells showed a dramatically increased proportion of CD25 expression (approx. 38\%) in contrast to T1/ST2- Th cells (approx. 8\%) (Figure 4a and $\mathbf{b}$ ). Increased CD25 expression on $\mathrm{T} 1 / \mathrm{ST} 2^{+}$Th cells is accompanied by enhanced CD44 expression pointing to an effector-like Th cell phenotype (Figure 4a). In addition, CD62L expression is hardly detectable on the $\mathrm{CD} 44^{+} /$ $\mathrm{CD} 25^{-}$and $\mathrm{CD} 44^{+} / \mathrm{CD} 25^{+} \mathrm{T} 1 / \mathrm{ST} 2^{+}$Th cell subsets (data not shown). Interestingly, the $\mathrm{T} 1 / \mathrm{ST} 2{ }^{+} \mathrm{CD} 25^{+} \mathrm{Th}$ cells account for $41 \%$ of all CD $25^{+}$Th cells in infected WT mice (Figure $4 \mathbf{b}$, for comparison see also Figure 3c, right panel; total number of 

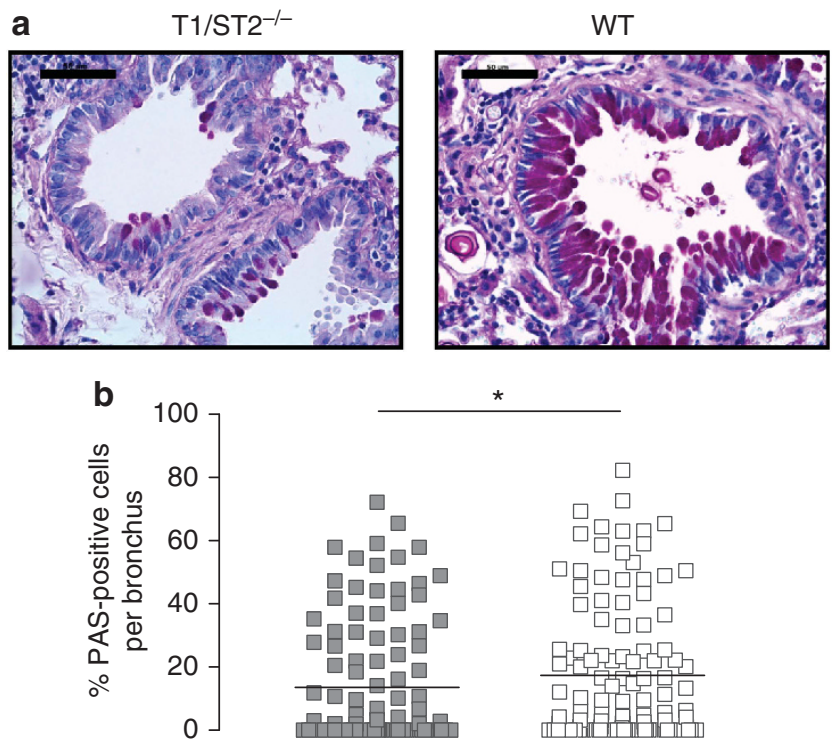

$\star$

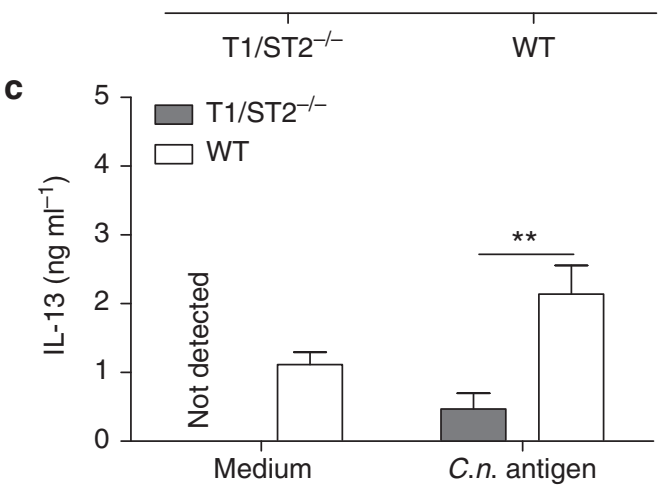

Figure 2 Interleukin (IL)-13-dependent mucus production in airways is decreased in the absence of T1/ST2. Wild-type (WT) and T1/ST2 ${ }^{-/-}$ mice were infected intranasally with Cryptococcus neoformans 1841 and analyzed 49 days post infection (dpi) ( $n=6$ mice per genotype and experiment). (a) Periodic acid-Schiff (PAS) staining was performed on lung sections from T1/ST2 ${ }^{-/-}$(left panel) or WT (right panel) mice. Bar $=50 \mu \mathrm{m}$. (b) PAS score (see Methods section) was calculated from counting PAS-positive epithelial cells of 10 bronchi of each mouse, e.g., as shown in a. (c) Purified lung leukocytes of two mice (of the same genotype) were pooled (i.e., 3 pools per genotype and experiment) and restimulated for $22 \mathrm{~h}$ with $C$. neoformans (C.n.) antigen or left untreated IL-13 was measured by enzyme-linked immunosorbent assay (ELISA) in culture supernatants. Data from two independent experiments were pooled and expressed as median or mean \pm s.e.m. Statistical analysis was performed using the Mann-Whitney $U$-test for PAS score and the Student's $t$-test for supernatant analysis. ${ }^{*} P \leq 0.05$ and ${ }^{* *} P \leq 0.01$.

Th cells: T1/ST2 ${ }^{+}, 1.4 \mathrm{E} 5 \pm 0.27 \mathrm{E} 5$; T1/ST2- ${ }^{-}$10.7E5 $\left.\pm 0.93 \mathrm{E} 5\right)$. In contrast to WT mice, $\mathrm{T} 1 / \mathrm{ST} 2^{-1-}$ mice show reduced proportions of $\mathrm{CD} 25^{+} \mathrm{Th}$ cells, and consequently harbor fewer $\mathrm{CD} 25^{+}$Th cells in total (Figure 4c). Moreover, it is remarkable that highly activated Th cells (i.e., $\mathrm{CD} 44^{+} / \mathrm{CD} 25^{-}$and $\mathrm{CD} 44^{+} / \mathrm{CD} 25^{+}$) not only show a higher proportion of $\mathrm{T} 1 / \mathrm{ST} 2^{+}$ cells (Figure 5a) but also an increased expression of T1/ST2 per cell indicated by elevated mean fluorescence intensity (MFI) (Figure 5b; correlation between MFI T1/ST2 and MFI CD25: $R=0.9766$ ), suggesting a higher sensitivity to IL-33. In summary, the data demonstrate that the degree of T1/ST2 expression positively correlates with the level of Th cell activation.

\section{T1/ST2 signaling predominantly impacts Th2-associated cytokine production}

Similar to IL-5 and IL-13, IL-4 is an important player in susceptibility against $C$. neoformans. ${ }^{10,11,30}$ The analysis of supernatants of antigen-restimulated pulmonary leukocytes revealed that IL-4 was likewise reduced as shown for IL-5 and IL-13 (Figures $\mathbf{1 b}$ and 2c), although the synthesis of IL-4 was not entirely dependent on T1/ST2 (Figure 6a). The difference in IL-4 production prompted us to analyze serum IgE. We found a reduction of serum IgE in the absence of T1/ST2, but this difference was at the border of statistical significance $(P=0.0526$ and data not shown). It was intriguing to test whether there is also an impact of T1/ST2 on Th subsets other than the Th2 subpopulation. Thus, we investigated Th1 and Th17 responses by analyzing the production of interferon- $\gamma$ (Figure 6b) and IL-17 (data not shown) by pulmonary leukocytes. Production of interferon- $\gamma$ as well as IL-17 did not differ between T1/ST2 ${ }^{-1-}$ and WT mice, indicating that Th1 and Th17 differentiation is not regulated by T1/ST2. This probably argues for a dominant role of Th2 cytokines over Th1 and Th17-related cytokines in pulmonary cryptococcosis. Finally, we analyzed the production of IL-10 that has been associated with immunopathology in cryptococcosis, ${ }^{8}$ and similar to the Th2 cytokines IL-4, IL-5, and IL-13, it was found to be significantly reduced in the absence of T1/ST2 (Figure 6c).

\section{T1/ST2 not only dictates the quantity but also the co-expression profile of the Th2 cytokines IL-4, IL-5, and} IL-13, resulting in loss of fungal control

As shown above, T1/ST2 expression on $\mathrm{CD}^{+}{ }^{+} \mathrm{T}$ cells during immunity to pulmonary infection with $C$. neoformans marks highly activated $\mathrm{Th} 2$ cells and profoundly influences the secretion of Th2 cytokines, in particular IL-5 and IL-13. Next, we wished to characterize directly the cellular source of the individual Th2 cytokines. We performed intracellular cytokine staining in Th cells that were isolated from lungs of infected T1/ST2 $2^{-1-}$ or WT mice and restimulated with cryptococcal antigen. For the selective analysis of Cryptococcus-induced Th cell responses, we made use of CD40L (CD154) expression. CD154 is only detectable in antigen-specific Th cells. ${ }^{31,32}$ To this end, we divided the Th cells of WT mice into T1/ST2 ${ }^{+}$and T1/ST2 ${ }^{-}$Th cells (for comparison see Figures $3 \mathbf{b}$ or $\mathbf{4 a}$ ) and analyzed the $\mathrm{CD} 154^{+}$Th subpopulations of each group. The same gating strategy was applied for Th cells from T1/ST2 ${ }^{-/-}$ mice. WT and $\mathrm{T} 1 / \mathrm{ST} 2^{-1-}$ mice did not differ in the percentage of CD154 $4^{+}$Th cells after restimulation (mean \pm s.e.m. of CD154 ${ }^{+}$ Th cells from WT and T1/ST2 ${ }^{-1-}$ mice: $3.027 \pm 0.4379 \%$ and $3.522 \pm 0.3925 \%$, respectively). As Th1 and Th17 cytokines were not affected (Figure $\mathbf{6 c}$ and data not shown), we focused on Th2 cytokines IL-4, IL-5, and IL-13. The magnitude of the MFI indicates the amount of the analyzed cytokine per cell. Therefore, T1/ST2 ${ }^{+}$Th cells show the greatest potential for each of the three investigated Th2 cytokines (Figure $7 \mathbf{a}$ and b). Interestingly, 

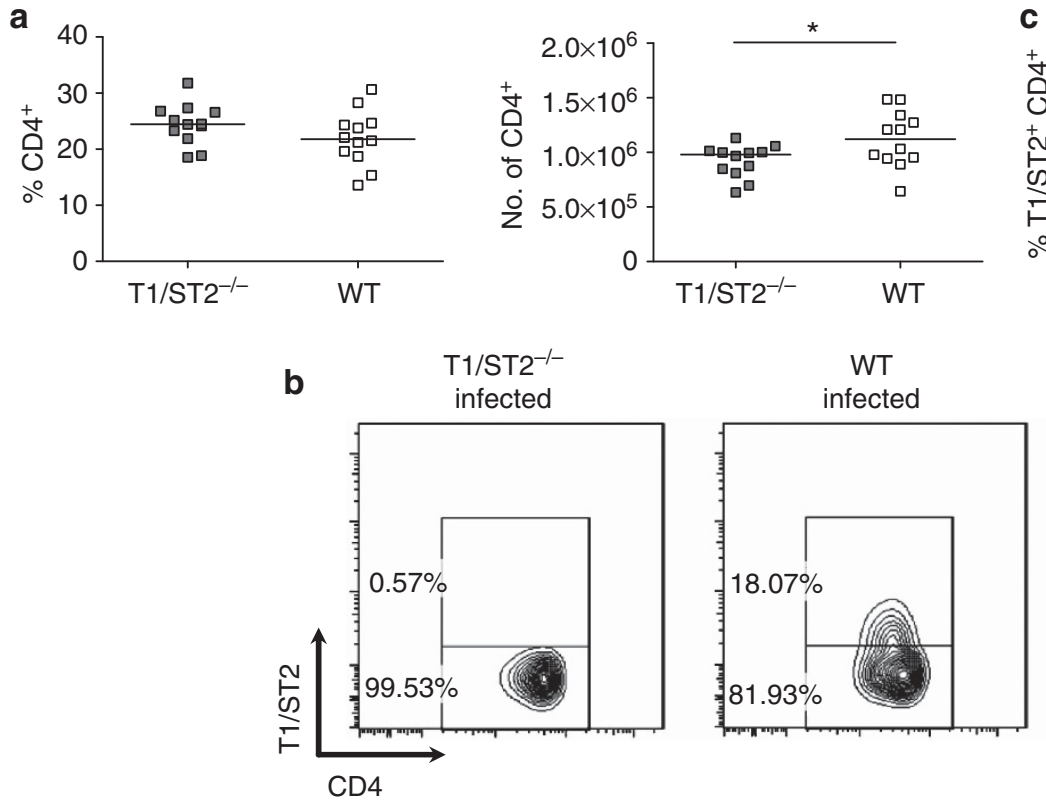
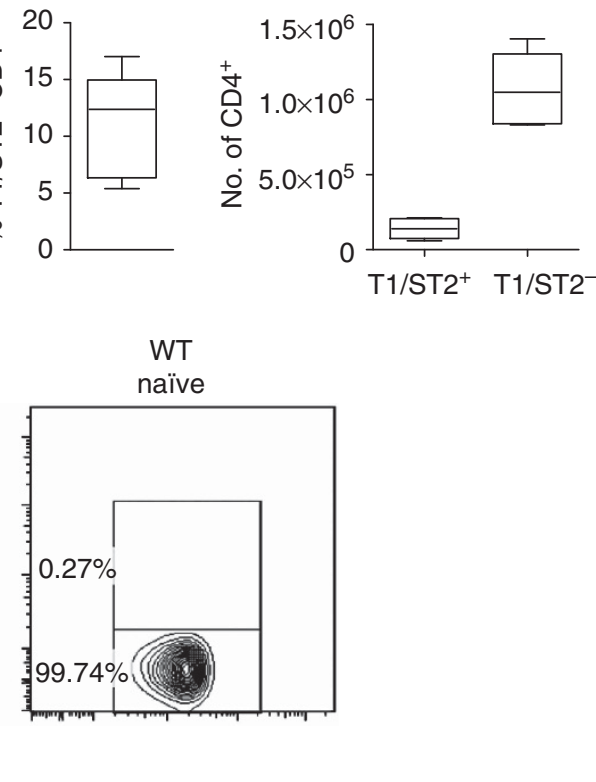

Figure 3 Intranasal infection with Cryptococcus neoformans induces T1/ST2 expression on pulmonary T helper (Th) cells. Wild-type (WT) and $\mathrm{T} 1 / \mathrm{ST} 2^{-/-}$mice were infected intranasally with $C$. neoformans 1841 and analyzed 49 days post infection (dpi) ( $n=6$ mice per genotype and experiment). (a) Relative (left panel) and total (right panel) numbers of CD4+ Th cells in lungs of WT and T1/ST2 ${ }^{-/-}$mice. (b) Representative fluorescence-activated cell sorting (FACS) plots of pulmonary CD4 ${ }^{+}$Th cells of infected T1/ST2 ${ }^{-/-}$(left panel), WT (middle panel), and naïve WT (right panel) mice. (c) Proportion of CD4 ${ }^{+} \mathrm{T} 1 / \mathrm{ST} 2^{+}$Th cells (left panel) and absolute numbers of CD4 ${ }^{+} \mathrm{T} 1 / \mathrm{ST} 2^{+}$, and T1/ST2 ${ }^{-} \mathrm{Th}$ cells (right panel) in WT mice. Data from two independent experiments were pooled and expressed as median or mean \pm s.e.m. Statistical analysis was performed using the Student's $t$-test. ${ }^{*} P \leq 0.05$.

the T1/ST2 ${ }^{-}$Th cells from WT mice closely resemble the Th cells from $\mathrm{T} 1 / \mathrm{ST} 2^{-1-}$ mice in terms of Th2 cytokine MFI (Figure 7a), indicating an independent mode of action of the $\mathrm{T} 1 / \mathrm{ST} 2^{+} \mathrm{Th} 2$ cells in WT mice. There was almost no bystander activity (i.e., cytokine-producing CD154- Th2 cells) detectable (data not shown).

Previously, studies designed to find a Th cell correlate of vaccine efficiency pointed to polyfunctional Th1 cells producing simultaneously interferon- $\gamma$, tumor necrosis factor- $\alpha$, and IL-2. ${ }^{31}$ In a very recent report, we described polyfunctional Th2 cells that mediate susceptibility in pulmonary cryptococcosis. ${ }^{28}$ We now wished to investigate the potential role of T1/ST2 for the production of polyfunctional Th2 cells. Therefore, proportions of single and multiple Th2 cytokine producers of the three groups were analyzed. A representative example is shown in Figure 8a. It is remarkable that IL-5 production is strongly dependent on T1/ST2 expression as there are almost no IL- $5^{+}$ Th cells detectable in T1/ST2 ${ }^{-}$Th cells from WT and T1/ST2 ${ }^{-/-}$ mice (Figure 8a). A similar result was seen for IL-13, although to a somewhat lesser degree, nicely reflecting the amounts of both cytokines found in the supernatants of restimulated pulmonary leukocytes (Figures $\mathbf{1} \mathbf{b}$ and $\mathbf{2} \mathbf{c}$ ). This demonstrates that indeed T1/ST2 ${ }^{+}$Th cells are major cellular sources of IL-5 and IL-13. T1/ST2 ${ }^{+}$Th cells contain relatively more cytokine producers and also bifunctional Th2 cytokine producers (i.e., IL-4/IL-5-, IL-4/IL-13-, IL-5/IL-13-producing cells) compared with WT T1/ST2- and T1/ST2-/- Th cells (Figure $8 \mathbf{b}$; left panel). Again, WT T1/ST2 ${ }^{-}$and $\mathrm{T} 1 / \mathrm{ST} 2^{-/-}$Th cells are com- parably low for cytokine production and Th2 cytokine polyfunctionality. Expanding the view on Th cells producing even all three Th2 cytokines simultaneously, the T1/ST2 ${ }^{+}$fraction represents the major source, whereas WT T1/ST2- and T1/ ST2 ${ }^{-1-}$ Th cells only show a minor degree of polyfunctionality (Figure 8b; right panel). The detection of residual IL-4, IL-13, and small amounts of IL- 5 in T1/ST2 ${ }^{-}$and T1/ST2 ${ }^{-/-}$Th cells indicate the presence of a distinct $\mathrm{Th} 2$ cell subpopulation that is of "lower" quality in contrast to T1/ST2 ${ }^{+} \mathrm{Th} 2$ cells. Taken together, the presence of T1/ST2 on Th cells identifies a subpopulation of highly activated Th2 cells, profoundly regulating not only the frequency of cytokine-producing Th cells but also their cytokine coexpression pattern in bronchopulmonary cryptococcosis with direct pathophysiological consequences (Figures 1a and 2a, b). As the control of fungal growth is central to protection, we determined lung burden of WT and T1/ $\mathrm{ST} 2^{-1-}$ mice (Figure 9). At 7 weeks after infection, T1/ST2 ${ }^{-1-}$ mice significantly harbor a lower fungal burden in their lungs showing better growth control of $C$. neoformans in the absence of T1/ST2-regulated Th2 cytokine expression. In conclusion, expression of T1/ST2 significantly contributes to the quantity and quality of Th2 cells, resulting in a defect of protective immunity against pulmonary cryptococcosis.

\section{DISCUSSION}

In this work, we investigated the function of the IL-33 receptor T1/ST2 in a mucosal infection model with an animal and human fungal pathogen. Th cells have a decisive role in the regu- 

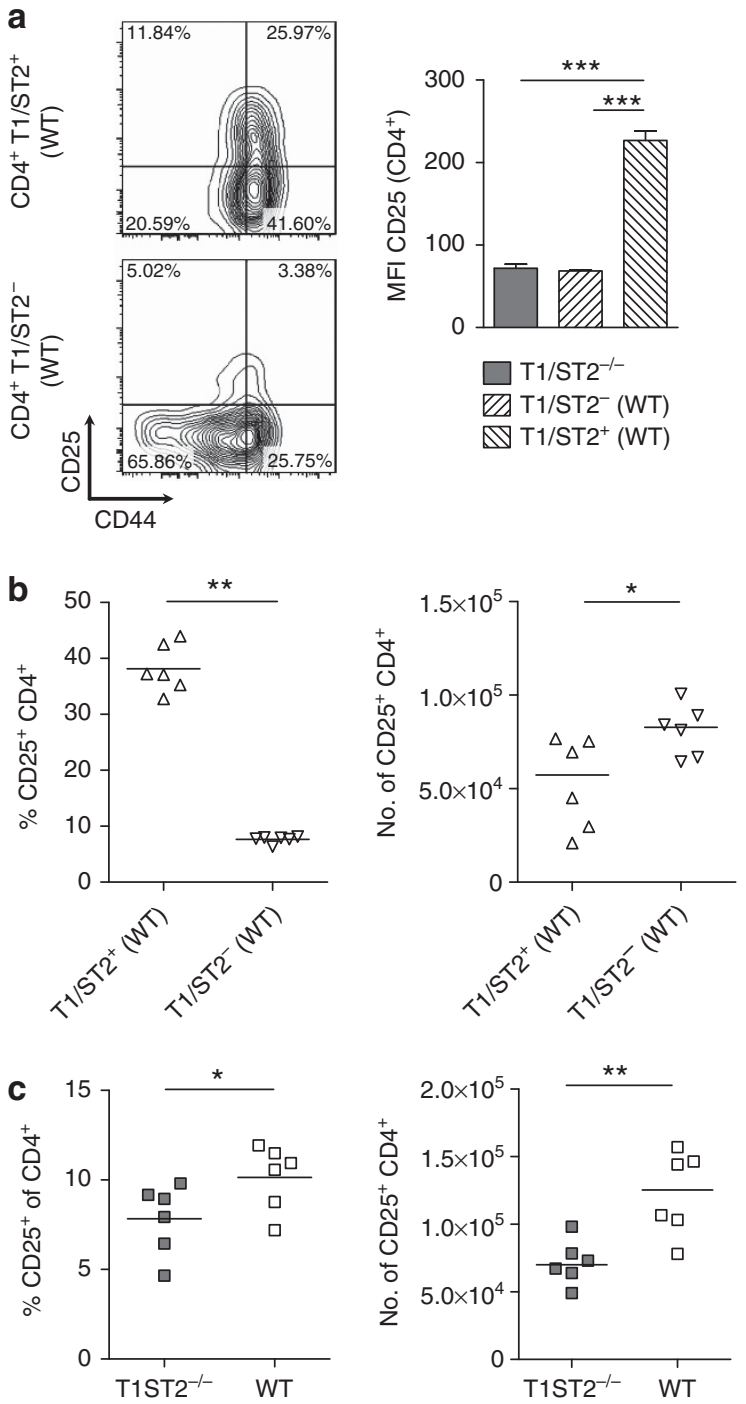

Figure $4 \mathrm{CD} 4{ }^{+} \mathrm{T} 1 / \mathrm{ST} 2^{+} \mathrm{T}$ helper $(\mathrm{Th})$ cells have a highly activated phenotype. Wild-type (WT) and T1/ST2 $2^{-/-}$mice were infected intranasally with Cryptococcus neoformans 1841 and analyzed 49 days post infection (dpi) ( $n=6$ mice per genotype and experiment).

(a) Representative fluorescence-activated cell sorting (FACS) plots (from one WT mouse) showing CD25/CD44 expression on pulmonary $\mathrm{T} 1 / \mathrm{ST} 2^{+}$(upper panel) and T1/ST2- (lower panel) CD4 ${ }^{+}$Th cells. CD25 mean fluorescence intensity (MFI) on CD4 ${ }^{+}$Th cells from WT ( $\mathrm{T} 1 / \mathrm{ST} 2^{+}$and $\mathrm{T}_{1 / \mathrm{ST}}{ }^{-}$) and $\mathrm{T} 1 / \mathrm{ST} 2^{-/-}$mice. (b) Comparison of relative CD25 expression on $\mathrm{T} 1 / \mathrm{ST} 2^{+}$and $\mathrm{T} 1 / \mathrm{ST} 2^{-} \mathrm{CD} 4^{+}$Th cells (left panel) and absolute numbers of $\mathrm{CD} 25^{+} \mathrm{T}_{1 / \mathrm{ST}} 2^{+}$and $\mathrm{CD} 25^{+} \mathrm{T} 1 / \mathrm{ST} 2^{-} \mathrm{CD} 4^{+}$ Th cells (right panel) from WT mice. (c) Comparison of relative CD25 expression on $\mathrm{CD}^{+}$Th cells (left panel) and absolute numbers of CD25+ CD $4^{+}$Th cells from T1/ST2 ${ }^{-/-}$and WT mice. Data from one of two independent experiments are shown and expressed as median or mean \pm s.e.m. Statistical analysis was performed using the Student's $t$-test for $\mathrm{CD} 25 \mathrm{MFI}$, total $\mathrm{CD}_{4}{ }^{+}, \mathrm{CD} 25^{+}$Th cells, and relative CD25 expression on $\mathrm{CD}^{+}$Th cells in WT vs. T1/ST2 ${ }^{-/-}$mice and Mann-Whitney $U$-test for relative CD25 expression on $\mathrm{T} 1 / \mathrm{ST} 2^{+}$and $\mathrm{T} 1 / \mathrm{ST} 2^{-} \mathrm{CD} 4^{+}$Th cells in WT mice. ${ }^{*} P \leq 0.05,{ }^{* \star} P \leq 0.01$, and ${ }^{* \star *} P \leq 0.001$.

lation of immunity against $C$. neoformans by either mediating protection via a Th1 response $\mathrm{e}^{24,30,33}$ or susceptibility via a Th2 response..$^{8,10,12,13}$ We studied the role of T1/ST2 signaling in
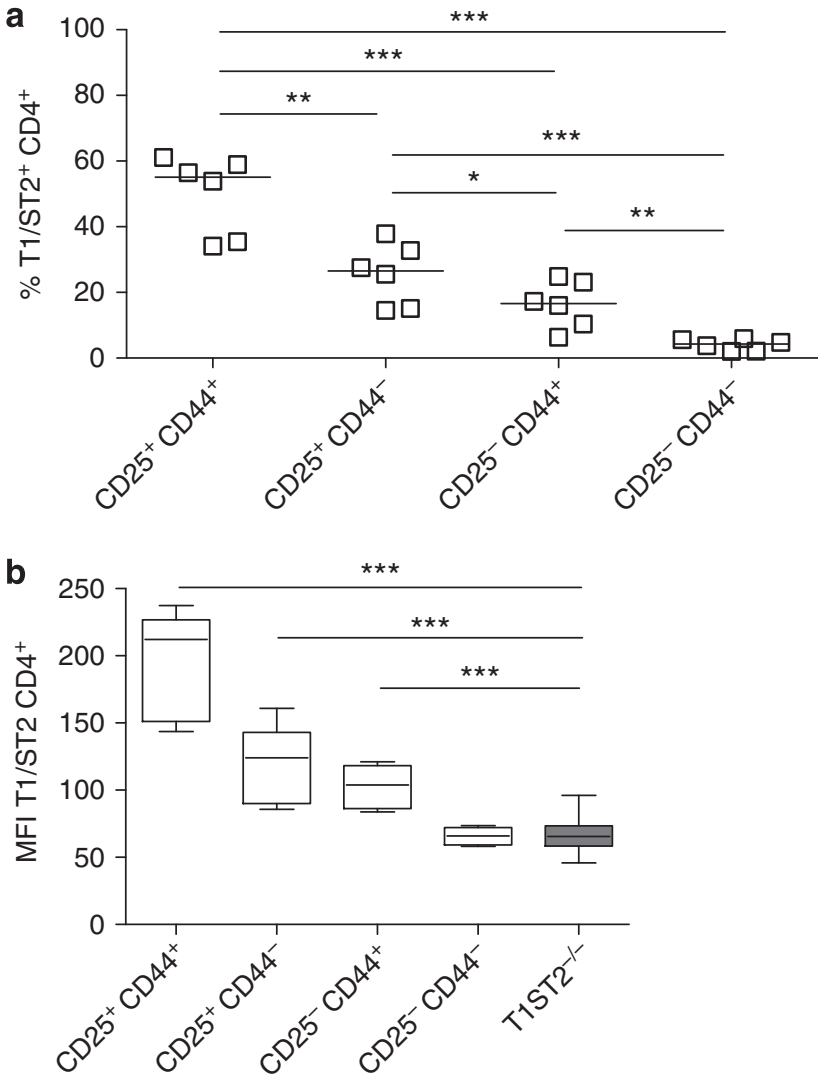

Figure 5 The majority of activated CD4 ${ }^{+}$T-helper (Th) cells expresses enhanced levels of T1/ST2 depending on the degree of activation. Wild-type (WT) and T1/ST2 ${ }^{-/-}$mice were infected intranasally with Cryptococcus neoformans 1841 and analyzed 49 days post infection (dpi) ( $n=6$ mice per genotype and experiment). (a) Proportions of T1/ST2 expression on several subsets of activated (dependent on CD25 and CD44 expression) pulmonary CD4 ${ }^{+}$Th cells from WT mice. (b) Mean fluorescence intensity (MFI) of T1/ST2 expression on subsets shown in (a). MFI of T1/ST2 on CD4 ${ }^{+} \mathrm{T} 1 / \mathrm{ST} 2^{-/-}$Th cells is shown as baseline in the T1/ST2 detecting channel. Data from one of two independent experiments are shown and expressed as median or mean \pm s.e.m. Statistical analysis was performed using the Student's $t$-test. ${ }^{*} P \leq 0.05$, ${ }^{* *} P \leq 0.01$, and ${ }^{* \star *} P \leq 0.001$.

Th cell activation and cytokine production during pulmonary cryptococcosis. First, we find upregulation of T1/ST2 expression on pulmonary Th cells upon intranasal infection with C. neoformans. Second, $\mathrm{T} 1 / \mathrm{ST} 2{ }^{+}$cells represent the majority of Th cells with an increased expression of CD44 and CD25. This points to the presence of a specialized subset of activated Th2 cells. ${ }^{34}$ The higher responsiveness for IL-2 by increased CD25 expression may provide better accessibility of the il4 locus in Th2 cells and allow for enhanced production of IL-4, IL-5, and IL-13 mRNA. ${ }^{35}$ Thus, IL-2 does not act solely as a growth factor for Th2 cells but also shapes type 2 cytokine production. Another study revealed STAT5 binding to the il5 and il13 loci in Th2 cells after stimulation with IL-2. ${ }^{36}$ Conversely, among the total activated Th cells, most show an increased T1/ST2 MFI that correlates with the degree of activation (CD25/CD44). Taken together, the strong correlation between T1/ST2 expression and Th2 activation suggests a novel mode of synergism between 

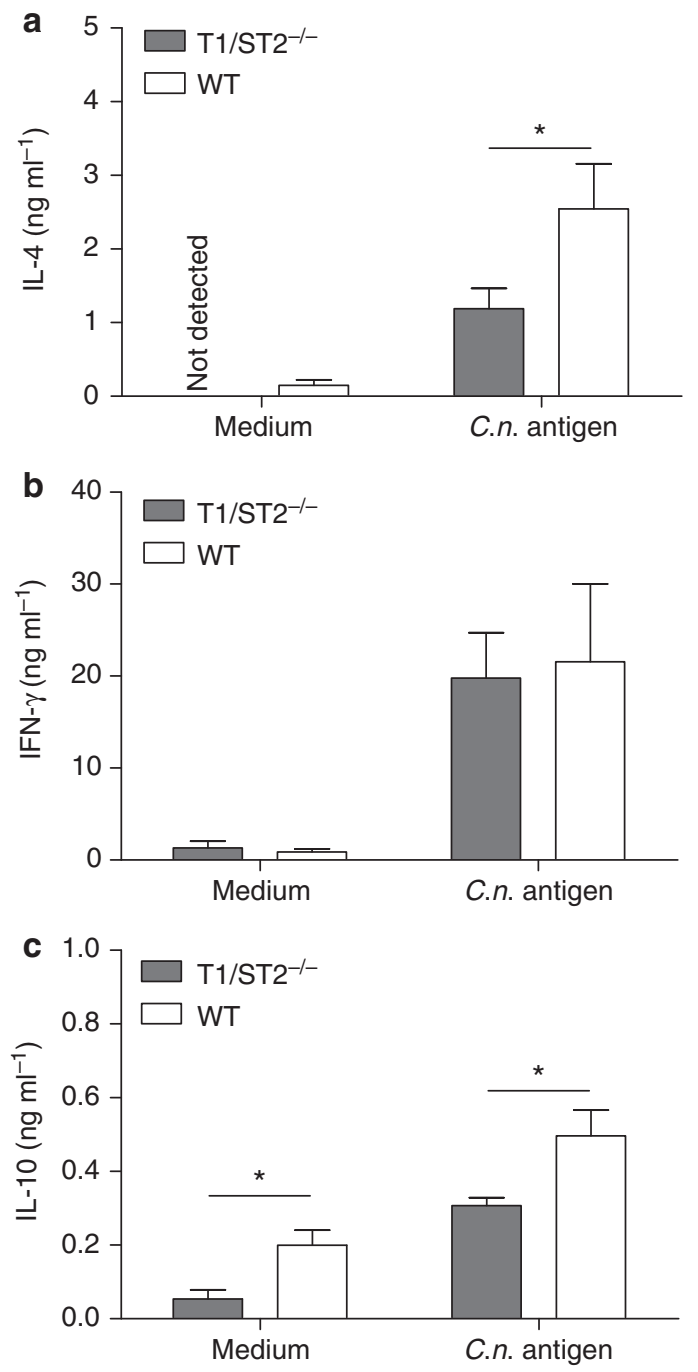

Figure 6 T1/ST2 regulates interleukin (IL)-4 and IL-10 but not interferon (IFN)- $\gamma$ production by pulmonary leukocytes. Wild-type (WT) and $\mathrm{T} 1 / \mathrm{ST} 2^{-/-}$mice were infected intranasally with Cryptococcus neoformans 1841 and analyzed 49 days post infection (dpi) ( $n=6$ mice per genotype and experiment). Isolated pulmonary leukocytes of two mice (of the same genotype) were pooled (i.e., 3 pools per genotype and experiment) and restimulated for $22 \mathrm{~h}$ with $C$. neoformans (C.n.) antigen or left untreated. (a) IL-4, (b) IFN- $\gamma$, and (c) IL-10 were measured by enzyme-linked immunosorbent assay (ELISA) in culture supernatant. Data from two independent experiments were pooled ( $=12$ mice per genotype, i.e., six subpools in total) and expressed as mean \pm s.e.m. Statistical analysis was performed using the Student's $t$-test. ${ }^{*} P \leq 0.05$.

IL-2 and IL-33 signaling in Th2 cells. Synergy between IL-2 and IL-33 could engage Th2 cells to develop to potent effector cells. Consistent with such a synergistic action of IL- 2 and IL-33 on type 2 cytokine production by T1/ST2 $2^{+}$Th cells, both cytokines are also known to induce T-cell proliferation. ${ }^{37,38}$

As we found higher percentages of $\mathrm{T} 1 / \mathrm{ST} 2^{+}$Th cells preferentially expressing CD44 and CD25 at the primary site of infection as compared with draining lymph nodes (data not shown), we extend data from an earlier report, which demonstrated that T1/ST2 expression is required for Th cells homing to the footpads of mice infected with Leishmania major. ${ }^{27}$ Homing
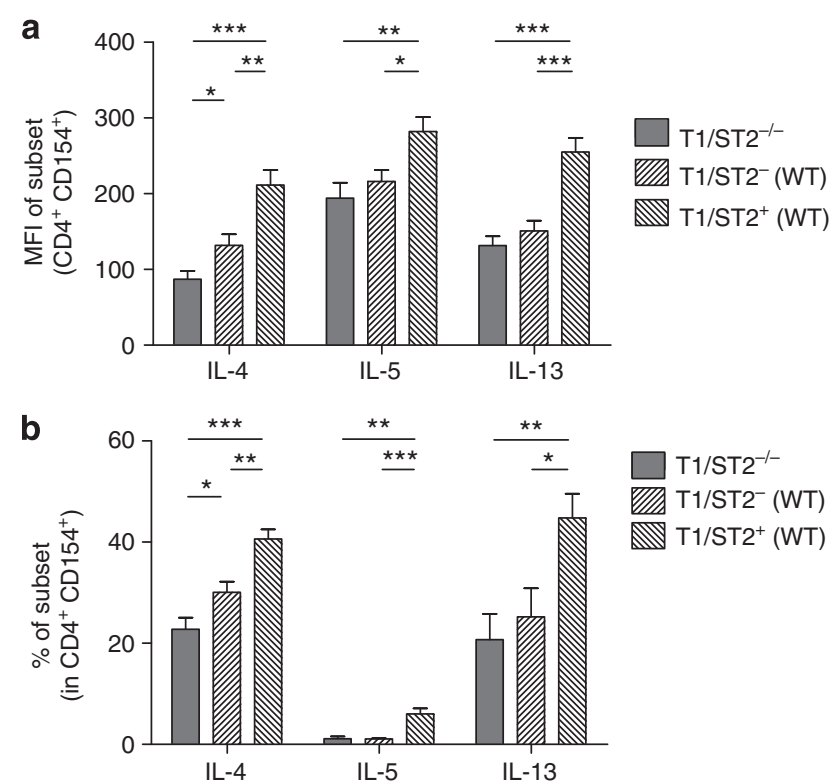

Figure $7 \mathrm{CD} 4^{+} \mathrm{T} 1 / \mathrm{ST} 2^{+} \mathrm{T}$-helper (Th) cells are potent Th2 cytokine producers. Wild-type (WT) and T1/ST2 ${ }^{-/-}$mice were infected intranasally with Cryptococcus neoformans 1841 and analyzed 49 days post infection (dpi) ( $n=6$ mice per genotype and experiment). Purified pulmonary leukocytes of two mice (of the same genotype) were pooled (i.e., 3 pools per genotype and experiment) and restimulated with C. neoformans antigen for $22 \mathrm{~h}$. (a) In antigen-specific (CD154 $\left.{ }^{+}\right) \mathrm{CD}^{+}$ Th cells from WT (either $\mathrm{T} 1 / \mathrm{ST} 2^{+}$or $\mathrm{T} 1 / \mathrm{ST} 2^{-}$) and $\mathrm{T} 1 / \mathrm{ST} 2^{-/-}$mice, mean fluorescence intensities (MFIs) of IL-4, IL-5, and IL-13 were determined by intracellular cytokine staining. (b) Same cells as in a were analyzed for the frequency of single cytokine producers. Data from two independent experiments were pooled (=12 mice per genotype, i.e., six subpools in total) and expressed as mean \pm s.e.m. Statistical analysis was performed using the Student's $t$-test for MFI. ${ }^{*} P \leq 0.05$, ${ }^{\star *} P \leq 0.01$, and ${ }^{\star \star *} P \leq 0.001$

of CD $44^{+} \mathrm{T} 1 / \mathrm{ST} 2{ }^{+}$Th cells to the effector site provides evidence for T1/ST2-dependent induction of Th cell effector status. The same authors hypothesize that T1/ST2 expression by Th cells may require the presence of high antigen load and chronic stimulation. ${ }^{27}$ Our model fulfills the requirement of a chronic disease with high antigen load at infection site and strengthens the idea of local T1/ST2 ${ }^{+}$Th cells as effector cells as we do not find remarkable CD62L expression.

In light of the activated phenotype of T1/ST2 ${ }^{+}$Th cells, we analyzed a potential correlation between T1/ST2 expression and cytokine production. Current research on Th cell cytokine production focuses on cytokine coexpression patterns, resulting in distinct subpopulations of Th cells. Thus, for Th1 cells coexpressing multiple cytokines at the single-cell level (termed polyfunctional Th cells), it was shown that these cells are not only more potent producers of any single cytokine but also good correlates of vaccine efficiency. ${ }^{31}$ It was intriguing to speculate that T1/ST2-dependent highly activated Th2 cells with polyfunctional Th2 cytokine expression could be detrimental in a disease setting such as pulmonary allergic inflammation. Indeed, T1/ST2 ${ }^{+}$Th cells show increased MFIs for IL-4, IL-5, and IL-13. Moreover, T1/ST2 ${ }^{+}$Th cells contain by far the highest 
a

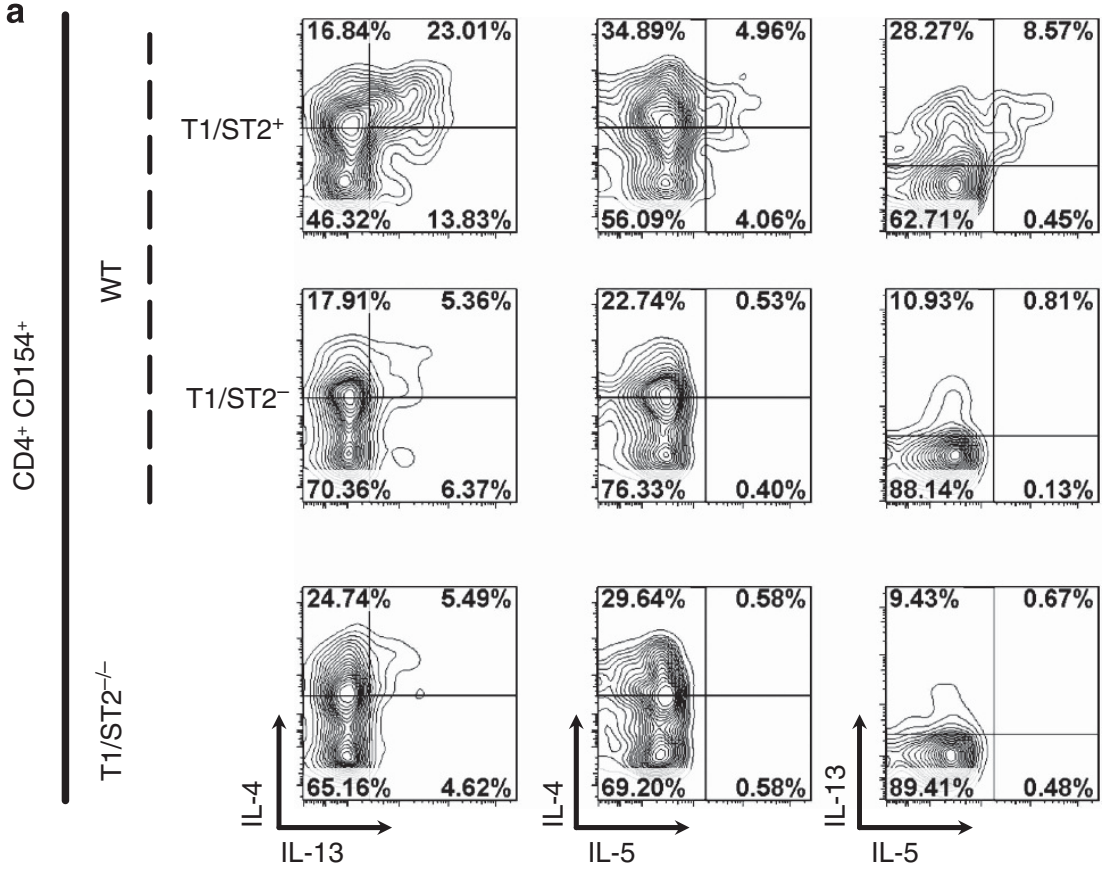

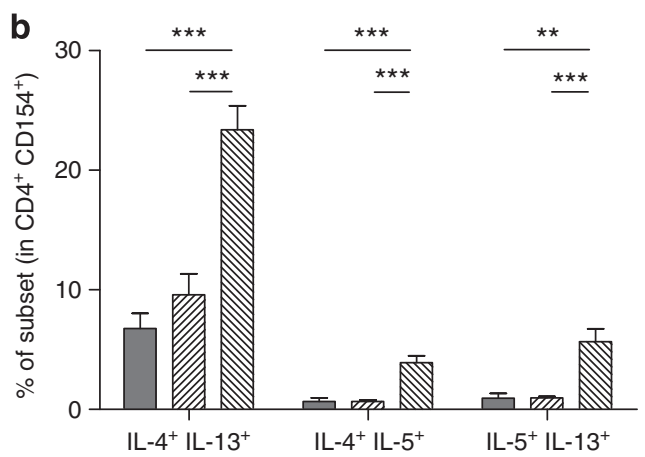

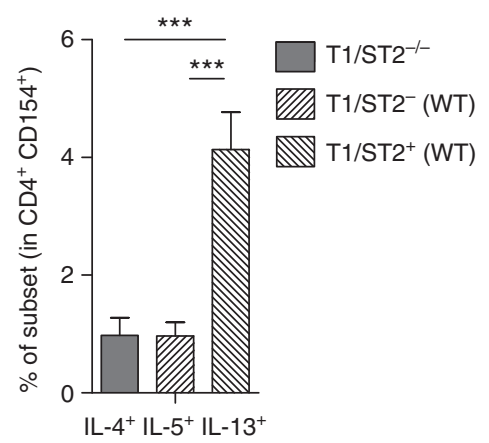

Figure 8 Antigen-specific T1/ST2+ CD4+ T-helper (Th) cells represent polyfunctional Th2 cytokine producers. Wild-type (WT) and T1/ST2-/- mice were infected intranasally with Cryptococcus neoformans 1841 and analyzed 49 days post infection (dpi) ( $n=6$ mice per genotype and experiment). Purified pulmonary leukocytes of two mice (of the same genotype) were pooled (i.e., 3 pools per genotype and experiment) and restimulated with C. neoformans antigen for $22 \mathrm{~h}$. (a) Representative fluorescence-activated cell sorting (FACS) plots of antigen-specific (CD154 ${ }^{+}$) CD4 ${ }^{+}$Th cells from WT (either $\mathrm{T}_{1 / \mathrm{ST} 2}{ }^{+}$or T1/ST2-) and T1/ST2 ${ }^{-/-}$mice producing interleukin (IL)-4, IL-5, and IL-13 are shown. (b) Same cells as in panel a were analyzed for the frequency of double cytokine producers (left panel). Double producers were analyzed for the production of the third cytokine, and the percentage of triple producers of WT (either $\mathrm{T} 1 / \mathrm{ST} 2^{+}$or $\mathrm{T} 1 / \mathrm{ST} 2^{-}$) and $\mathrm{T} 1 / \mathrm{ST} 2^{-/-}$mice was calculated. Data from two independent experiments (=12 mice per genotype, i.e., six subpools in total) were pooled and expressed as mean \pm s.e.m. Statistical analysis was performed using the Student's t-test. ${ }^{* *} P \leq 0.01$ and ${ }^{* \star *} P \leq 0.001$.

frequency of double and triple Th2 cytokine producers (Figure 8b) responsible for higher type 2 cytokine production and elevated immunopathology in WT as compared with $\mathrm{T} 1 / \mathrm{ST} 2^{-1-}$ mice (Figure $7 \mathbf{b}$ ). This allowed us to link the expression of T1/ST2 on Th cells, in particular with IL-5 as well as IL-13 production (Figure 8a), and therefore extends previous findings referring to IL-5 only. ${ }^{39}$ Instead, IL- 4 is less controlled by T1/ST2 as shown here (Figure 8a) and previously by Guo et al. ${ }^{40} \mathrm{IL}-4$ production by Th2 cells appears to depend on other regulatory elements such as the type I IL-4R as published recently by us for pulmonary cryptococcosis. ${ }^{11,28}$

There are previous findings on T1/ST2 pointing to pathogen-dependent differences. In a L. major model, the usage of a T1/ST2-depleting antibody showed an improved Th1 response, while there was no influence on Th2-mediated pathology. It is remarkable that the authors did not find an attenuation of disease under these neutralizing conditions. Yet, they still find Th2 cells identified by IL- 4 and IL- 10 production. ${ }^{41}$ We agree that T1/ST2 is not essential for Th2 differentiation as we also find IL-4-producing Th cells in Cryptococcus-infected T1/ST2 $2^{-/-}$ mice (Figures 7 and 8). With a pronounced impact of T1/ST2 on the production of IL- 5 and IL-13, it appears that IL-5 and IL-13 may have a greater role in cryptococcosis than in L. major infection. Another report negating a role for T1/ST2 in allergic airway inflammation also relies on the analysis of IL-4 synthesis, but IL- 5 and IL-13 may have been more informative under the experimental settings used in this report. ${ }^{42}$ Therefore, it may be more adequate to define the function of T1/ST2 as 


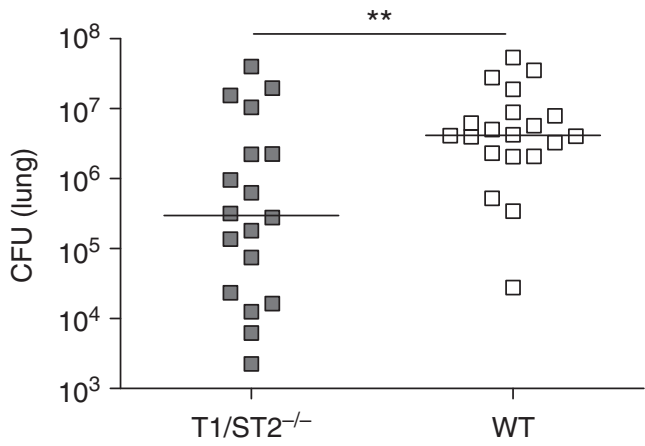

Figure 9 In the absence of T1/ST2 signaling, fungal growth is enhanced at the primary site of infection. Wild-type and T1/ST2 ${ }^{-/-}$mice were infected intranasally with Cryptococcus neoformans 1841 and analyzed 49 days post infection (dpi). Serial dilutions of digested lung tissue were plated on Sabouraud dextrose agar plates and colonyforming-units (CFU) of individual mice were calculated. Data from three independent experiments (6-8 mice per experiment, i.e., $18-20$ mice in total) were pooled and expressed as mean \pm s.e.m. Statistical analysis was performed using the Mann-Whitney $U$-test. ${ }^{* *} P \leq 0.01$.

a regulator of Th2 cell quality, which reflects coexpression of multiple Th2 cytokines in single cells termed polyfunctional Th2 cells. In pulmonary cryptococcosis, we find T1/ST2-dependent polyfunctional Th2 cells to be linked with pulmonary allergic inflammation and a defect in pulmonary control of fungal infection. Thus, in addition to the IL-4R on Th cells, ${ }^{28} \mathrm{~T} 1 / \mathrm{ST} 2$ is another regulator of Th2 cell polyfunctionality driving the disease progression during pulmonary cryptococcosis.

Our data show that T1/ST2 is a marker of Th2 cell activation and substantially regulates type 2 cytokine production. Transfer experiments with cytokine-expressing $\mathrm{T} 1 / \mathrm{ST} 2{ }^{+} \mathrm{CD} 154^{+} \mathrm{Th}$ cells would be interesting to study their stability and further development. Unfortunately, this approach is currently technically not feasible as staining of cytokines and CD154 requires fixation with permeabilization and is therefore not applicable on living cells. Moreover, it would be also of great interest to study the effect of T1/ST2 on Th2 cell quality in humans with Th2-biased diseases. Taken together, we provide a novel mechanism by which the cytokine receptor T1/ST2 induces complex Th2 cytokine profiles in an in vivo model of bronchopulmonary mycosis. This may allow for novel therapeutic strategies targeting IL-33 and its receptor. Recently, for Aspergillus fumigatus infection attenuation of fungal asthma was achieved using neutralizing antibodies against membrane-bound T1/ST2 ${ }^{6}$

Determination of the expression of T1/ST2 and CD25/CD44 on Th cells may be utilized for monitoring disease severity in Th2-driven allergic inflammation. The underlying basis is provided by our study showing that highest expression of T1/ST2 is found on $\mathrm{CD} 25^{+} \mathrm{CD} 44^{+}$Th cells (Figure $\mathbf{5 b}$ ) and T1/ST2 expression is associated with highest $\mathrm{Th} 2$ cytokine production at a single-cell level (Figure 7a). Furthermore, T1/ST2 ${ }^{+}$Th cells appear to be the dominant sources of the effector cytokines IL-5 and IL-13 (Figures $\mathbf{1 b}, \mathbf{2 c}$, and 8a). It will be interesting to analyze the contribution of other Th2-associated cytokines such as IL- $25^{43}$ and TSLP ${ }^{44}$, which might be responsible for the residual
Th2 cytokine production in T1/ST2 ${ }^{-1-}$ mice (Figures $\mathbf{1 b}, \mathbf{2 c}$, and $\mathbf{8 a}$ ).

IL-13 is known to induce potently mucus production in goblet cells in the lung ${ }^{45}$ and IL-5 is tightly linked to the differentiation and activation of eosinophilic granulocytes. ${ }^{46}$ As we previously reported, IL-13-dependent mucus production and airway hyperreactivity are cardinal features of chronic pulmonary cryptococcosis. ${ }^{10,11}$ However, it was unclear what the driving factor of IL-5 and IL-13 synthesis is. We now report that the absence of T1/ST2 is the reason for a drastic reduction of IL-5 and IL-13 at the primary site of infection, resulting in strong reduction of mucus production in bronchial epithelium as well as much lesser recruitment of eosinophilic granulocytes that are of importance as IL-4 producers. ${ }^{13}$ Recent reports from parasite models point to $\mathrm{T} 1 / \mathrm{ST} 2{ }^{+}$innate immune cells as significant cellular sources of IL-5 and IL-13. ${ }^{47-50}$ Early IL-5- and IL-13-producing innate cells may act before the Th2-cell-derived IL-4 response commences 6 weeks after intranasal infection with C. neoformans. ${ }^{13}$ Ongoing experiments already demonstrate that IL-5 and IL-13 precede the surprisingly late IL-4 production in cryptococcosis (manuscript in preparation).

\section{METHODS}

Mice. The 8- to 10-week-old female wild-type (WT) (Janvier, Le Genest Saint Isle, France) and T1/ST2 ${ }^{-1-}$ mice on BALB/c background ${ }^{21}$ were maintained in an IVC-caging system under specific pathogen-free conditions and in accordance with the guidelines approved by the Animal Care and Usage Committee of the "Landesdirektion Leipzig." Sterile food and water were given ad libitum. The mice were tested periodically for pathogens in accordance with the recommendations for health monitoring of mice provided by the Federation of European Laboratory Animal Science Associations (FELASA) accreditation board. All mice were tested negative for pinworms and other endo- and ectoparasites.

C. neoformans and infection of mice. The clinical isolate C. neoformans strain 1841 (serotype D) was derived from an HIV patient. ${ }^{24}$ It was kept as a frozen stock in $10 \%$ skim milk and was grown in Sabouraud dextrose medium ( $2 \%$ glucose, $1 \%$ peptone; Sigma Aldrich, Munich, Germany) overnight on a shaker at $30^{\circ} \mathrm{C}$. Cells were harvested and washed twice with sterile phosphate-buffered saline (PBS), and counted in a hematocytometer. Inocula were finally diluted in PBS to a concentration of $2.5 \times 10 \mathrm{E} 4 \mathrm{ml}^{-1}$ for intranasal infection. Mice received $20 \mu \mathrm{l}$ containing 500 colony-forming units. Before infection, mice were anesthetized by an intraperitoneal injection with a 1:1 mixture of ketamine $\left(100 \mathrm{mg} \mathrm{ml}^{-1}\right.$; Ceva Tiergesundheit, Düsseldorf, Germany) and xylazine $\left(20 \mathrm{mg} \mathrm{ml}^{-1}\right.$; Ceva Tiergesundheit).

Preparation and stimulation of pulmonary leukocytes, determination of fungal lung burden. Infected mice were monitored daily for survival and morbidity. For preparation of pulmonary leukocytes, lungs were perfused using sterile $0.9 \%$ sodium chloride solution. After removal, lungs were minced and digested for $30 \mathrm{~min}$ at $37^{\circ} \mathrm{C}$ in RPMI 1640 (with $10 \%$ heat-inactivated fetal bovine serum (FBS) and 1\% penicillin/ streptomycin) supplemented with collagenase $\left(0.689 \mathrm{mg} \mathrm{ml}^{-1}\right.$; Roche Diagnostics Deutschland GmbH, Mannheim, Germany), $10 \mu \mathrm{M}$ sodium pyruvate, and DNase IV (111 $\mathrm{U} \mathrm{ml}^{-1}$; Sigma Aldrich). Following passage through a $100 \mu \mathrm{m}$ nylon mesh (BD Biosciences, Heidelberg, Germany), the filtrate was resuspended in $1 \mathrm{ml}$ PBS containing 3\% FBS (Invitrogen, Darmstadt, Germany) and $50 \mu \mathrm{l}$ were taken for colony-forming units enumeration. Serial dilutions were plated on Sabouraud dextrose agar plates and colonies were counted after an incubation period of $48 \mathrm{~h}$ at $30^{\circ} \mathrm{C}$. With the remaining cells, lysis of red blood cells was carried out with Gey's 
solution. Afterwards, cells were washed with PBS containing 3\% heatinactivated FBS, resuspended in 70\% Percoll (GH Healthcare Biosciences AB, Uppsala, Sweden), and layered under 30\% Percoll. After centrifugation, leukocytes were recovered from interphase, washed with Iscove's modified Dulbecco's medium (PAA Laboratories, Cölbe, Germany), and counted in Trypan blue (Fluka Chemie AG, Buchs, Switzerland). For surface staining, $2 \times 10 \mathrm{E} 5$ cells were used, and for intracellular cytokine staining, at least $1 \times 10 \mathrm{E} 6$ cells were acquired. Purified cells were adjusted to $5 \times 10 \mathrm{E} 6 \mathrm{ml}^{-1}$ in Iscove's modified Dulbecco's medium (with $10 \% \mathrm{FBS}$ and $1 \%$ penicillin/streptomycin) and stimulated $22 \mathrm{~h}$ with specific antigen or left unstimulated. The acapsular C. neoformans serotype D strain CAP67 (kindly provided by B. Fries Albert-Einstein College of Medicine, Bronx, NY, USA) was used as a specific stimulus $(1 \times 10 \mathrm{E} 7$ cryptococci per ml, termed "C.n. antigen") for restimulation of pulmonary leukocytes from C. neoformans-infected mice. It was cultured and maintained in the same manner as strain 1841 and before use heat-inactivated at $60^{\circ} \mathrm{C}$ for $1 \mathrm{~h} .{ }^{51}$ For blocking of cytokine secretion, brefeldin A $\left(5 \mu \mathrm{g} \mathrm{ml}^{-1}\right.$; Sigma Aldrich) was added for the last $4 \mathrm{~h}$.

Flow cytometry. LIVE/DEAD Fixable Aqua Dead Cell Stain Kit (Invitrogen) was used to ensure discrimination and exclusion of dead cells during analysis. For surface staining, cells were incubated for $15 \mathrm{~min}$ on ice with FcR block $(0.1 \mu \mathrm{g} / 1 \times 10 \mathrm{E} 6$ cells; purified from $2.4 \mathrm{G} 2$ hybridoma supernatant) and rat serum (Sigma Aldrich) to avoid unspecific binding of antibody fluorochrome conjugates. Subsequently, staining antibodies were added for a 30-min incubation on ice. For the characterization of surface antigens, dead cells, doublets, and debris were excluded, followed by plotting of CD11c against Siglec-F to analyze eosinophilic granulocytes as SSC ${ }^{\text {hi }}, \mathrm{CD} 11 \mathrm{c}^{-/ \mathrm{dim}}$, and Siglec- $\mathrm{F}^{+}$cells. The expression of T1/ST2, CD44, and CD25 on Th cells was carried out by gating on $\mathrm{CD}^{+}$lymphocytes (FSC $\left.{ }^{\text {low }} / \mathrm{SSC}^{\mathrm{low}}\right)$. For intracellular cytokine staining, cells were fixed with $2 \%$ paraformaldehyde (Serva, Heidelberg, Germany) upon application of LIVE/DEAD Fixable Aqua Dead Cell Stain Kit (Invitrogen) for $20 \mathrm{~min}$ on ice. Permeabilization of cells was achieved using fluorescence-activated cell sorting (FACS) buffer (i.e., PBS containing $3 \%$ heat-inactivated FBS and $0.1 \%$ sodium azide) containing $0.5 \%$ saponin $\left(\mathrm{wv}^{-1}\right.$; Serva). After permeabilization, cells were incubated for 15 min on ice with FcR block $(0.1 \mu \mathrm{g} / 1 \times 10 \mathrm{E} 6$ cells; purified from $2.4 \mathrm{G} 2$ hybridoma supernatant) and rat serum (Sigma Aldrich) in FACS buffer containing $0.5 \%$ saponin to avoid unspecific staining. Afterwards, staining antibodies were added in FACS buffer containing $0.5 \%$ saponin for a 30-min incubation on ice. The analysis of intracellular cytokines was based on the following gating strategy:

First, dead cells, doublets, and debris were excluded. Second, CD4 was plotted against CD40L (CD154). CD4/CD154 double-positive cells were used for further analysis of T1/ST2 expression in WT mice. In T1/ST2 ${ }^{-/-}$ mice, all CD4 ${ }^{+} \mathrm{CD} 154^{+}$were used as one population. For analysis of overall frequency of the producers of IL-4, IL-5, and IL-13 including mean fluorescence intensity (MFI), histograms were created for each cytokine. For analysis of frequency of double cytokine producers, all possible combinations of IL-4, IL-5, and IL-13 were plotted. Cells positive for two of the three cytokines were analyzed. Finally, double producers were analyzed for the third cytokine (e.g., IL- $4^{+} / \mathrm{IL}-5^{+}$population was analyzed for IL-13). Frequencies were calculated by normalizing each subpopulation of CD $154^{+}$Th cells to $100 \%$ (i.e., T1/ST2 ${ }^{+}$and T1/ST2 ${ }^{-}$ $\mathrm{CD}_{154^{+}}{ }^{+}$Th cells in WT mice and all CD154 ${ }^{+}$Th cells in T1/ST2 $2^{-1-}$ mice). Antibodies and FcR block for intracellular staining were diluted in FACS buffer containing $0.5 \%$ saponin $\left(\mathrm{wv}^{-1}\right.$; Serva). For specific stainings, the following antibodies were used: anti-CD4-APC-Cy7 (GK1.5; BD Biosciences), anti-IL-4-PE-Cy7 (BVD6-24G2; ebioscience, Frankfurt, Germany), anti-IL-5-APC (TRFK5; BioLegend, Fell, Germany), anti-IL13-PE (eBio13A; ebioscience), anti-IL-17-PacificBlue (TC11-18H10.1; BioLegend), anti-Siglec-F-PE (E50-2440; BD Biosciences), antiCD11c-APC (N418; ebioscience) anti-CD154-PE (MR1; ebioscience), anti-CD25-Biotin (PC61.5; ebioscience), anti-CD44-PacificBlue (IM7; BioLegend), anti-CD62L-PE-Cy7 (MEL-14; BioLegend), anti-CCR6PE (29-2L17; BioLegend), anti-CXCR3-APC (CXCR3-173; BioLegend),
anti-F4/80-PE-Cy7 (BM8; ebioscience), and anti-T1/ST2-FITC (DJ8; MD Biosciences, Zürich, Switzerland). Appropriate isotype antibodies were from ebiosciences and BioLegend. Cells labeled with biotinylated antibodies were further stained with streptavidin-PerCp-Cy5.5 (ebiosciences). Cells were aquired on a BD FACS LSR II at the Core Unit Fluorescence Technologies of the IZKF Leipzig using DIVA version 6.1.1 and FlowJo version 7.6.1 (Treestar, Ashland, OR, USA) software was used for analysis.

Cytokine enzyme-linked immunosorbent assays. Cytokine concentrations were determined by sandwich enzyme-linked immunosorbent assay systems. To determine the concentration of IL-4, monoclonal antibody (mAb) 11B11 was used as the capture Ab and biotin-labeled BVD6-24G2 (BD Biosciences) was used as the detection Ab. IL-5 measurement was carried out using the mAb TRFK5 (BD Biosciences) for capturing and the biotinylated $\mathrm{mAb}$ TRFK4 (BD Biosciences) for detection. IL-10 was measured using a murine IL-10 ELISA Development Kit (Peprotech, Hamburg, Germany). For IL-13 and IL-17, R\&D Systems Duoset kits (R\&D Systems GmbH, Wiesbaden, Germany) were used. Before development of enzyme-linked immunosorbent assays, using biotinylated detection antibodies, incubation with peroxidase-labeled streptavidin (Southern Biotechnology Associates, Birmingham, AL, USA) was carried out. IFN- $\gamma$ was captured by $\mathrm{mAb} A N-18$ and detected by peroxidase-labeled mAb XMG1.2. Development was done using the TMB 2-Component Microwell Peroxidase Substrate Kit (KPL, Gaithersburg, MD).

Histopathology. Lung samples were processed for histological analysis as described previously. ${ }^{13}$ The percentage of PAS-positive goblet cells in bronchial tissue was determined by an independent investigator by counting PAS-positive and PAS-negative bronchial epithelial cells of 5 cross-sections of proximal bronchi of two slices per lung (different lung regions), with a total of 10 cross sections per mouse. This is modified from Fulkerson et al. ${ }^{52}$ and termed PAS score in this manuscript.

Statistical evaluation. The Mann-Whitney $U$-test was performed for ex vivo recruitment analysis and phenotyping of pulmonary leukocytes, PAS score, colony-forming units enumeration, and analysis of cytokine coexpression. The $t$-test was used for the analysis of cytokine in supernatants, and MFI. Data are presented as either medians or means \pm s.e.m. The level of confidence for significance is indicated as follows: ${ }^{\star} P \leq 0.05$; ${ }^{* *} P \leq 0.01$; and ${ }^{* * *} P \leq 0.001$. Correlation coefficient between the MFIs of T1/ST2 and CD25 was tested with Pearson's correlation test.

\section{ACKNOWLEDGMENTS}

We thank Dr McKenzie for agreeing to work with $\mathrm{T} 1 / \mathrm{ST} 2^{-/-}$mice. We also thank Dr Drube for providing T1/ST2 ${ }^{-/-}$mice and Dr Kamradt for critical reading of the manuscript. We are grateful to $A$. Grohs and P. Krumbholz for superb technical assistance, and R. Voigtländer and S. Leitenroth for keeping the mice during the experiments. We also acknowledge the help and advice by Dr. A Lösche and K Jäger with multiparameter FACS analysis using the LSR II at the Core Unit Fluorescence Technologies of the IZKF Leipzig. Finally, we also thank C. Westermann for counting bronchoepithelial cells allowing the calculation of the PAS score.

This project was funded by grant AL 371/5-4 (to GA) by the Deutsche Forschungsgemeinschaft (German Research Foundation).

\section{DISCLOSURE}

The authors declared no conflict of interest.

C) 2013 Society for Mucosal Immunology

\section{REFERENCES}

1. Loures, F.V., Pina, A., Felonato, M. \& Calich, V.L. TLR2 is a negative regulator of Th17 cells and tissue pathology in a pulmonary model of fungal infection. J. Immunol. 183, 1279-1290 (2009).

2. Stuehler, C. et al. Cross-protective TH1 immunity against Aspergillus fumigatus and Candida albicans. Blood 117, 5881-5891 (2011). 
3. Wuthrich, M., Deepe, G. \& Klein, B. Adaptive immunity to fungi. Annu. Rev. Immunol. 30, 115-148 (2012).

4. Doherty, T.A. et al. Alternaria induces STAT6-dependent acute airway eosinophilia and epithelial FIZZ1 expression that promotes airway fibrosis and epithelial thickness. J. Immunol. 188, 2622-2629 (2012).

5. Goldman, D.L. et al. Increased chitinase expression and fungal-specific antibodies in the bronchoalveolar lavage fluid of asthmatic children. Clin. Exp. Allergy 42, 523-530 (2012).

6. Ramaprakash, H. et al. Targeting ST2L potentiates CpG-mediated therapeutic effects in a chronic fungal asthma model. Am. J. Pathol. 179, 104-115 (2011).

7. Park, B.J. et al. Estimation of the current global burden of cryptococcal meningitis among persons living with HIV/AIDS. AIDS 23, 525-530 (2009).

8. Hernandez, Y. et al. Distinct roles for IL-4 and IL-10 in regulating T2 immunity during allergic bronchopulmonary mycosis. J. Immunol. 174, 1027-1036 (2005)

9. Huffnagle, G.B., Boyd, M.B., Street, N.E. \& Lipscomb, M.F. IL-5 is required for eosinophil recruitment, crystal deposition, and mononuclear cell recruitment during a pulmonary Cryptococcus neoformans infection in genetically susceptible mice (C57BL/6). J. Immunol. 160, 2393-2400 (1998).

10. Muller, U. et al. IL-13 induces disease-promoting type 2 cytokines, alternatively activated macrophages and allergic inflammation during pulmonary infection of mice with Cryptococcus neoformans. J. Immunol. 179, 5367-5377 (2007).

11. Muller, U. et al. A gene-dosage effect for interleukin-4 receptor alpha-chain expression has an impact on Th2-mediated allergic inflammation during bronchopulmonary mycosis. J. Infect. Dis. 198, 1714-1721 (2008).

12. Stenzel, W. et al. IL-4/IL-13-dependent alternative activation of macrophages but not microglial cells is associated with uncontrolled cerebral cryptococcosis. Am. J. Pathol. 174, 486-496 (2009).

13. Piehler, D. et al. Eosinophils contribute to IL-4 production and shape the T-helper cytokine profile and inflammatory response in pulmonary cryptococcosis. Am. J. Pathol. 179, 733-744 (2011).

14. Carriere, V. et al. IL-33, the IL-1-like cytokine ligand for ST2 receptor, is a chromatin-associated nuclear factor in vivo. Proc. Natl. Acad. Sci. USA 104, 282-287 (2007)

15. Schmitz, J. et al. IL-33, an interleukin-1-like cytokine that signals via the $\mathrm{IL}-1$ receptor-related protein ST2 and induces Thelper type 2-associated cytokines. Immunity 23, 479-490 (2005).

16. Zaiss, M.M. et al. IL-33 shifts the balance from osteoclast to alternatively activated macrophage differentiation and protects from TNF-alphamediated bone loss. J. Immunol. 186, 6097-6105 (2011).

17. Granne, I. et al. ST2 and IL-33 in pregnancy and pre-eclampsia. PLoS One 6, e24463 (2011)

18. Nelson, M.P. et al. IL-33 and M2a alveolar macrophages promote lung defense against the atypical fungal pathogen Pneumocystis murina. J. Immunol. 186, 2372-2381 (2011).

19. Kouzaki, H., lijima, K., Kobayashi, T., O'Grady, S.M. \& Kita, H. The danger signal, extracellular ATP, is a sensor for an airborne allergen and triggers IL-33 release and innate Th2-type responses. J. Immunol. 186, 4375-4387 (2011).

20. Kondo, Y. et al. Administration of IL-33 induces airway hyperresponsiveness and goblet cell hyperplasia in the lungs in the absence of adaptive immune system. Int. J. Immunol. 20, 791-800 (2008).

21. Townsend, M.J., Fallon, P.G., Matthews, D.J., Jolin, H.E. \& McKenzie, A.N. T1/ST2-deficient mice demonstrate the importance of T1/ST2 in developing primary T helper cell type 2 responses. J. Exp. Med. 191, 1069-1076 (2000)

22. Wynn, T.A. IL-13 effector functions. Annu. Rev. Immunol. 21, 425-456 (2003).

23. Rothenberg, M.E. \& Hogan, S.P. The eosinophil. Annu. Rev. Immunol. 24, 147-174 (2006).

24. Decken, K. et al. Interleukin-12 is essential for a protective Th1 response in mice infected with Cryptococcus neoformans. Infect. Immun. 66, 4994-5000 (1998).

25. Walzl, G. et al. Inhibition of T1/ST2 during respiratory syncytial virus infection prevents Thelper cell type 2 (Th2)- but not Th1-driven immunopathology. J. Exp. Med. 193, 785-792 (2001).

26. Lohning, M. et al. T1/ST2 is preferentially expressed on murine Th2 cells, independent of interleukin 4, interleukin 5, and interleukin 10, and important for Th2 effector function. Proc. Natl. Acad. Sci. USA 95, 6930-6935 (1998).

27. Kropf, P., Bickle, Q., Herath, S., Klemenz, R. \& Muller, I. Organ-specific distribution of $\mathrm{CD} 4+\mathrm{T} 1 / \mathrm{ST} 2+\mathrm{Th} 2$ cells in Leishmania major infection. Eur. J. Immunol. 32, 2450-2459 (2002).
28. Muller, U. et al. Lack of IL-4 receptor expression on Thelper cells reduces Thelper 2 cell polyfunctionality and confers resistance in allergic bronchopulmonary mycosis. Mucosal Immunol. 5, 299-310 (2012).

29. Lohning, M. et al. T1/ST2 expression is enhanced on CD4+ T cells from schistosome egg-induced granulomas: analysis of Th cell cytokine coexpression ex vivo. J. Immunol. 162, 3882-3889 (1999).

30. Kawakami, K. et al. IL-18 contributes to host resistance against infection with Cryptococcus neoformans in mice with defective IL-12 synthesis through induction of IFN-gamma production by NK cells. J. Immunol. 165, 941-947 (2000)

31. Darrah, P.A. et al. Multifunctional TH1 cells define a correlate of vaccinemediated protection against Leishmania major. Nat. Med. 13, 843-850 (2007).

32. Frentsch, M. et al. Direct access to CD4+ T cells specific for defined antigens according to CD154 expression. Nat. Med. 11, 1118-1124 (2005).

33. Herring, A.C., Lee, J., McDonald, R.A., Toews, G.B. \& Huffnagle, G.B. Induction of interleukin-12 and gamma interferon requires tumor necrosis factor alpha for protective T1-cell-mediated immunity to pulmonary Cryptococcus neoformans infection. Infect. Immun. 70, 2959-2964 (2002).

34. Willerford, D.M. et al. Interleukin-2 receptor alpha chain regulates the size and content of the peripheral lymphoid compartment. Immunity 3 , 521-530 (1995).

35. Cote-Sierra, J. et al. Interleukin 2 plays a central role in Th2 differentiation. Proc. Natl. Acad. Sci. USA 101, 3880-3885 (2004).

36. Liao, W. et al. Priming for Thelper type 2 differentiation by interleukin 2-mediated induction of interleukin 4 receptor alpha-chain expression. Nat. Immunol. 9, 1288-1296 (2008).

37. Meisel, C. et al. Regulation and function of T1/ST2 expression on CD4+ T cells: induction of type 2 cytokine production by T1/ST2 cross-linking. J. Immunol. 166, 3143-3150 (2001)

38. Gillis, S., Ferm, M.M., Ou, W. \& Smith, K.A. T cell growth factor: parameters of production and a quantitative microassay for activity. J. Immunol. 120, 2027-2032 (1978)

39. Kurowska-Stolarska, M. et al. IL-33 induces antigen-specific IL-5+ T cells and promotes allergic-induced airway inflammation independent of IL-4. J. Immunol. 181, 4780-4790 (2008).

40. Guo, L. et al. IL-1 family members and STAT activators induce cytokine production by Th2, Th17, and Th1 cells. Proc. Natl. Acad. Sci. USA 106, 13463-13468 (2009).

41. Kropf, P., Herath, S., Klemenz, R. \& Muller, I. Signaling through the T1/ST2 molecule is not necessary for Th2 differentiation but is important for the regulation of type 1 responses in nonhealing Leishmania major infection. Infect. Immun. 71, 1961-1971 (2003).

42. Hoshino, K. et al. The absence of interleukin 1 receptor-related T1/ST2 does not affect $T$ helper cell type 2 development and its effector function. J. Exp. Med. 190, 1541-1548 (1999).

43. Fort, M.M. et al. IL-25 induces IL-4, IL-5, and IL-13 and Th2-associated pathologies in vivo. Immunity 15, 985-995 (2001).

44. Al-Shami, A., Spolski, R., Kelly, J., Keane-Myers, A. \& Leonard, W.J. A role for TSLP in the development of inflammation in an asthma model. J. Exp. Med. 202, 829-839 (2005).

45. Grunig, G. et al. Requirement for IL-13 independently of IL-4 in experimental asthma. Science 282, 2261-2263 (1998).

46. Tominaga, A. et al. Transgenic mice expressing a B cell growth and differentiation factor gene (interleukin 5) develop eosinophilia and autoantibody production. J. Exp. Med. 173, 429-437 (1991)

47. Moro, K. et al. Innate production of $T(H) 2$ cytokines by adipose tissueassociated c-Kit(+)Sca-1(+) lymphoid cells. Nature 463, 540-544 (2010).

48. Neill, D.R. et al. Nuocytes represent a new innate effector leukocyte that mediates type-2 immunity. Nature 464, 1367-1370 (2010).

49. Price, A.E. et al. Systemically dispersed innate IL-13-expressing cells in type 2 immunity. Proc. Natl. Acad. Sci. USA 107, 11489-11494 (2010).

50. Saenz, S.A. et al. IL25 elicits a multipotent progenitor cell population that promotes $T(H) 2$ cytokine responses. Nature 464, 1362-1366 (2010).

51. Kleinschek, M.A. et al. IL-23 enhances the inflammatory cell response in Cryptococcus neoformans infection and induces a cytokine pattern distinct from IL-12. J. Immunol. 176, 1098-1106 (2006).

52. Fulkerson, P.C. et al. A central regulatory role for eosinophils and the eotaxin/CCR3 axis in chronic experimental allergic airway inflammation. Proc. Natl. Acad. Sci. USA 103, 16418-16423 (2006). 